\title{
Modified gravity one-loop partition function
}

\author{
Shahab Shahidi ${ }^{1, \mathrm{a}}$, Farid Charmchi ${ }^{2, \mathrm{~b}}$, Zahra Haghani ${ }^{1, \mathrm{c}}$, Leila Shahkarami ${ }^{1, \mathrm{~d}}$ \\ ${ }^{1}$ School of Physics, Damghan University, Damghan 41167-36716, Iran \\ 2 School of Particles and Accelerators, Institute for Research in Fundamental Sciences (IPM), P.O. Box 19395-5531, Tehran, Iran
}

Received: 2 June 2018 / Accepted: 8 October 2018 / Published online: 17 October 2018

(C) The Author(s) 2018

\begin{abstract}
The one-loop partition function of the $f(R$, $R_{\mu \nu} R^{\mu \nu}$ ) gravity theory is obtained around $\mathrm{AdS}_{4}$ background. After a suitable choice of the gauge condition and computation of the ghost determinant, we obtain the one-loop partition function of the theory. The traced heat kernel over the thermal quotient of the $\mathrm{AdS}_{4}$ space is also computed and the thermal partition function is obtained for this theory. We then consider quantum corrections to the thermodynamical quantities in some special cases.
\end{abstract}

\section{Contents}

1 Introduction . . . . . . . . . . . . 1

2 The model . . . . . . . . . . . . . . . 2

3 The one-loop partition function of $f\left(R, R_{\mu \nu} R^{\mu \nu}\right)$ gravity ................ 3

4 The traced heat kernel on $\mathrm{AdS}_{4} \ldots \ldots \ldots$

5 Thermodynamical implications . . . . . . . . . 7

6 Conclusions and final remarks . . . . . . . . . . 8

A: Derivation of Eq. (3.8) _ . . . . . . . . . . . 9

B: Operators in the second order variation of the action . . 9

C: The masses . . . . . . . . . . . . . . . . 9

D: The decomposition determinants $\ldots \ldots \ldots$

References . . . . . . . . . . . . . . . . . 10

\section{Introduction}

At present, there is a large number of indications that Einstein's general relativity might be modified at the cosmological scales. At large scales, i.e., the regime of low energies, the introduction of some dark energy component seems to

\footnotetext{
a e-mail: s.shahidi@du.ac.ir

be-mail: charmchi@ipm.ir

ce-mail: z.haghani@du.ac.ir

de-mail: 1.shahkarami@du.ac.ir
}

be necessary to explain the accelerated expansion of the universe [1-4].

The interesting fact about the Einstein's gravity is that at UV limit the theory should also be modified, since it cannot explain the microscopic physics. The quantization of the Einstein-Hilbert action leads to a nonrenormalizable theory which is known for decades [5-8]. This suggests that we cannot quantize Einstein's general relativity along the path of the ordinary quantum field theory. One can think about the possibility of modifying the dynamics of the space-time in a nonlinear manner in such a way that the theory becomes renormalizable $[9,10]$. Perhaps the simplest possibility is to promote the Ricci scalar in the Einstein-Hilbert action to an arbitrary function of the Ricci scalar (the $f(R)$ theories of gravity). The quantum corrections to the $f(R)$ theories of gravity have been extensively investigated in the literature (see e.g. [11-15]).

The other possibility of modifying Einstein's gravity is to break the Lorentz symmetry of the theory and then add some spatial-derivative self-interaction terms in such a way that the resulting theory becomes renormalizable. This HoravaLifshitz quantum gravity theory [16] has been found to be very interesting both in theoretical and phenomenological point of view [17-21]. Also some attempts have been made to make the theory Lorentz-invariant [22-24].

Historically, the first example of a modified theory of gravity which is renormalizable at quantum level has been introduced in [25] in which the action

$S=\int d^{4} x \sqrt{-g}\left(\kappa^{2} R+a R^{2}+b R_{\mu \nu} R^{\mu \nu}\right)$,

is proven to be renormalizable for some special choices of the parameters. The beta function is also obtained in this case. This suggests that one can promote the Ricci scalar in the Einstein's theory to an arbitrary function of $R$ and $R_{\mu \nu} R^{\mu \nu}$, and even $R_{\mu \nu \rho \sigma} R^{\mu \nu \rho \sigma}$ (which is not necessary in 4D due to the Gauss-Bonnet theorem). Quantum corrections 
to the generalized Gauss-Bonnet theory over the de Sitter background are investigated in [26].

In this paper, we consider the $f\left(R, R_{\mu \nu} R^{\mu \nu}\right)$ gravity theory over the anti-de Sitter (AdS) background. The choice of this Lagrangian is interesting because it can be seen as a generalization of some interesting modified gravity theories including $f(R)$ gravity, conformal gravity and also Stelle's original work (one should however note that the $f(G)$ modified gravity theories where $G$ is the Gauss-Bonnet term cannot in general be expressed in our formalism). Also, the AdS background is interesting specially as a part of the AdS/CFT correspondence [27]. In this context, one of the most important quantities which should be computed is the partition function. The partition function of the gravity theory in the AdS background is then equivalent to the partition function of its dual conformal field theory.

In this work we compute the one-loop partition function of the $f\left(R, R_{\mu \nu} R^{\mu \nu}\right)$ theory around the $\mathrm{AdS}_{4}$ background. In order to do this, we fix the gauge in such a way that the resulting gauge-fixed Lagrangian becomes diagonal in the perturbation variables. Such a gauge fixing results in the introduction of the third ghost as discussed in [28]. Considering the ghost determinants and the Jacobian of the field transformation, we arrive at the one-loop partition function of the theory. One should note that the procedure introduced in this paper is a little bit different from the one usually done in the literature. We then discuss how we can recover the standard results in some special cases, e.g., in the case of Einstein's theory, first calculated in [29] where they consider the quantum structure of the quadratic theory of gravity on different backgrounds.

Using the heat kernel method, we are able to evaluate the determinants appearing in the partition function and compute the traced heat kernel over the thermal quotient of the $\mathrm{AdS}_{4}$ space [30,31], to obtain the thermal partition function in $f\left(R, R_{\mu \nu} R^{\mu \nu}\right)$ theory. The traced heat kernel over thermal quotient of the AdS space in the conformal gravity theory is discussed in [32]. We then use this method to obtain thermodynamical quantities such as Helmholtz free energy and also the entropy which should be viewed as a one-loop quantum correction to the entropy. We furthermore compare the consequences of the quantum corrections to the thermodynamical quantities in some special cases of interest.

\section{The model}

Let us consider the following general theory containing a function of both the Ricci scalar and the square of the Ricci tensor

$S=\int d^{4} x \sqrt{-g} f\left(R, R_{\mu \nu} R^{\mu \nu}\right)$, where $f$ is an arbitrary function with mass dimension 4. For our purposes, we need to calculate the second variation of this action on shell by imposing the equation of motion, coming from the first variation. By varying once the above action with respect to the metric tensor, one may obtain

$\delta S=\int d^{4} x \sqrt{-g} B_{\mu \nu} \delta g^{\mu \nu}$,

where $B_{\mu \nu}$ is the equation of motion which can be written as

$$
\begin{aligned}
B_{\mu \nu}= & f_{R} R_{\mu \nu}-\frac{1}{2} f g_{\mu \nu}+2 f_{X} R_{\alpha \mu} R_{\nu}^{\alpha}+\square H_{\mu \nu} \\
& +\nabla_{\alpha} \nabla_{\beta} H^{\alpha \beta} g_{\mu \nu}-2 \nabla_{\alpha} \nabla_{(\mu} H_{\nu)}^{\alpha}=0
\end{aligned}
$$

Here $H_{\mu \nu}=f_{R} g_{\mu \nu}+2 f_{X} R_{\mu \nu}$ and we have defined $X \equiv$ $R_{\mu \nu} R^{\mu \nu}, f_{R} \equiv \partial f / \partial R$ and $f_{X} \equiv \partial f / \partial X$.

It can be easily seen that the above equation acquires $\mathrm{AdS}_{4}$ solution. As we know, for the $\mathrm{AdS}_{4}$ space we have in general

$$
\bar{R}_{\mu \nu \rho \sigma}=\frac{\Lambda}{3}\left(\bar{g}_{\mu \rho} \bar{g}_{\nu \sigma}-\bar{g}_{\mu \sigma} \bar{g}_{\nu \rho}\right), \quad \bar{R}_{\mu \nu}=\Lambda \bar{g}_{\mu \nu}, \quad \bar{R}=4 \Lambda .
$$

Here and in the following few equations, we use the bar sign to denote the background metric and specially the $\mathrm{AdS}_{4}$ background which we are most interested in as a sample background in this paper. Using the above relations, the equation of motion (2.3) is reduced to

$2 f_{X} \Lambda^{2}+f_{R} \Lambda-\frac{1}{2} f=0$,

which can be solved for $\Lambda$ if one fixes the function $f$. For example, for the simple case $f=\kappa^{2} R+\eta R R_{\mu \nu} R^{\mu \nu}$ one has an $\mathrm{AdS}_{4}$ solution with $\Lambda=-\sqrt{\kappa^{2} / 4 \eta}$. This in fact means that the AdS background is the solution of the theory only for some special values of the parameters, including the cosmological constant. One should note that if the theory under consideration does not have an AdS solution, the corresponding one-loop effective action will be gauge-dependent. In the following we choose special forms of $f\left(R, R_{\mu \nu} R^{\mu \nu}\right)$ in which the AdS background is the solution of the theory.

Now, we proceed to find the second variation of the action. Consider the small fluctuation $h_{\mu \nu}$ of the tensor metric $g_{\mu \nu}$ around the background metric $\bar{g}_{\mu \nu}$ as follows

$g_{\mu \nu}=\bar{g}_{\mu \nu}+h_{\mu \nu}$.

Varying the relation (2.2) and evaluating it on shell for the background metric $\bar{g}_{\mu \nu}$, we arrive at 


$$
\begin{aligned}
\delta^{(2)} S= & \int d^{4} x \sqrt{-\bar{g}}\left(\delta \bar{B}^{\mu \nu} h_{\mu \nu}+\bar{B}^{\mu v} \delta h_{\mu \nu}\right. \\
& \left.-\frac{1}{2} \bar{g}_{\alpha \beta} \bar{B}_{\mu \nu} h^{\alpha \beta} h^{\mu \nu}\right) \\
= & \int d^{4} x \sqrt{-\bar{g}} \delta \bar{B}^{\mu \nu} h_{\mu \nu} .
\end{aligned}
$$

Again tensors with the bar sign are calculated in the background metric. The last two terms in the first line vanish by imposing the equation of motion (2.3).

Our task in the next section is to obtain the one-loop partition function for the theory of our interest. We use the definition $\mathcal{L}_{2} \equiv \delta \bar{B}^{\mu v} h_{\mu \nu}$ in what follows, for brevity of notation. We also omit the bar sign in the following sections, since all the calculations are performed in the background metric.

\section{The one-loop partition function of $f\left(R, R_{\mu \nu} R^{\mu \nu}\right)$ gravity}

Let us now compute the one-loop partition function of the gravitational theory (2.1). The partition function at the oneloop level can be obtained from the path integral over all fluctuations around the background $\left(h_{\mu \nu}\right)$ as

$$
Z^{1 \text { loop }}=\int D\left[h_{\mu \nu}\right] e^{-\delta^{(2)} S} .
$$

Because our theory is generally covariant, it has a gauge freedom of the form

$\delta h_{\mu \nu}=\nabla_{\mu} \epsilon_{\nu}+\nabla_{\nu} \epsilon_{\mu}$,

in which $\epsilon_{\mu}$ denote the parameters of the gauge group. In order to calculate the integral (3.1) only over physical degrees of freedom among all the ones that are related to each other through the gauge transformation (3.2), we have to fix the above gauge freedom. Consider the following gauge condition

$J_{\mu} \equiv G_{\mu \nu}\left(\xi^{\nu}-l^{\nu}\right)=0$.

Here $l^{\mu}$ is some arbitrary function and

$\xi_{\mu}=\nabla_{\alpha} h_{\mu}^{\alpha}-\left(\rho+\frac{1}{4}\right) \nabla_{\mu} h$,

where $h$ denotes the trace of $h_{\mu \nu}$, and the operator $G^{\mu \nu} \hat{a} \breve{A} \check{N}$ is defined as

$G^{\mu \nu}=\alpha\left(-g^{\mu \nu} \square-\gamma \nabla^{\mu} \nabla^{v}+\nabla^{\nu} \nabla^{\mu}\right)+\Lambda g^{\mu \nu}$,

in which $\alpha$ and $\gamma$ are some constants and $\Lambda$ is the $\operatorname{AdS}_{4}$ parameter. Note that the above gauge condition is different from the ordinary Lorentz condition because we are dealing with higher order gravity theories with fourth order derivative terms in the action. In this regard, $G_{\mu \nu}$ has the most general form for $f\left(R, R_{\mu \nu} R^{\mu \nu}\right)$ theory which makes the resulting gauge-fixed action diagonal [28]. Note that the expression (3.4) reduces to the Lorentz condition for $\rho=\frac{1}{4}$.

To impose the gauge-fixing condition on the functional integral (3.1), we insert the following expression into the action (3.1)

$$
\begin{aligned}
1 & =\int D\left[\epsilon_{\mu}\right] \delta\left(J_{\mu}\right) \operatorname{det}\left(\frac{\delta J^{\alpha}}{\delta \epsilon^{\beta}}\right) \\
& =\operatorname{det}^{-1} G_{\alpha \beta} \operatorname{det} M_{\alpha \beta} \int D\left[\epsilon_{\mu}\right] \delta\left(\xi^{\mu}-l^{\mu}\right),
\end{aligned}
$$

where the determinant in the first line is the Jacobian of the gauge transformation. In the second line of the above equation, we have used the relation $\delta\left(G_{\mu \nu}\left(\xi^{\nu}-l^{\nu}\right)\right)=$ $\operatorname{det}^{-1} G_{\alpha \beta} \delta\left(\xi^{\mu}-l^{\mu}\right)$ and defined

$M_{\beta}^{\alpha} \equiv \frac{\delta J^{\alpha}}{\delta \epsilon^{\beta}}=G_{\mu}^{\alpha} \frac{\delta \xi^{\mu}}{\delta \epsilon^{\beta}}$.

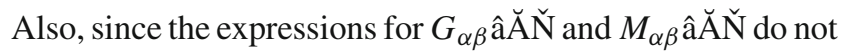
depend on the gauge parameters $\epsilon^{\mu}$, we put them outside the integral. The second part of the multiplication in the righthand side of the Eq. (3.7) can be calculated as (see appendix A for details)

$\frac{\delta \xi^{\mu}}{\delta \epsilon^{\beta}}=\delta_{\beta}^{\mu} \square+R_{\beta}^{\mu}+\left(\frac{1-4 \rho}{2}\right) \nabla^{\mu} \nabla_{\beta}$.

Inserting the obtained identity in the Eq. (3.1), the one-loop partition function becomes

$Z^{1 \text {-loop }}=\operatorname{det}^{-1} G_{\alpha \beta} \int D\left[h_{\mu \nu}\right] \operatorname{det} M_{\alpha \beta} \mathrm{e}^{-\int d^{4} x \sqrt{-g} \mathcal{L}_{2}} \delta\left(\xi^{\mu}-l^{\mu}\right)$.

Note that the integral over the gauge parameter $\epsilon$ gives us the volume of the gauge group which can be absorbed in the normalization of the partition function. Therefore, we have ignored it from the above expression.

Now, following the Faddeev-Popov proposal (see for example [33]), since in the gauge condition (3.3), $l^{\mu} \hat{\text { âA }} \check{\mathrm{N}}$ is arbitrary and the theory is invariant under the gauge transformation, we integrate the one-loop partition function (3.9) over $l^{\mu}$ with a normalized Gaussian weighting function as

$\operatorname{det}^{1 / 2} G_{\alpha \beta} \int D\left[l_{\mu}\right] e^{-\frac{1}{2} \int d^{4} x \sqrt{-g}\left(l^{\alpha} G_{\alpha \beta} l^{\beta}\right)}$,

where the determinant is the normalization factor of the Gaussian integral. Multiplying (3.9) by the above unity and using the delta function to perform the integral over $l_{\mu}$, we 
arrive at the following expression for the one-loop partition function

$$
Z^{1 \text { loop }}=\operatorname{det}^{-1 / 2} G_{\alpha \beta} \int D\left[h_{\mu \nu}\right] \operatorname{det} M_{\alpha \beta} e^{-\int d^{4} x \sqrt{-g}\left(\mathcal{L}_{2}+\mathcal{L}_{g f}\right)},
$$

where we have defined the gauge fixing Lagrangian as

$\mathcal{L}_{g f} \equiv \frac{1}{2} \xi_{\mu} G^{\mu \nu} \xi_{v}$.

We can substitute det $M_{\alpha \beta}$ in (3.11) with a functional integral as in the following form

$\operatorname{det} M_{\alpha \beta}=\int D\left[C_{\mu}\right] D\left[\bar{C}_{\mu}\right] e^{-\frac{1}{2} \int d^{4} x \sqrt{-g}\left(\bar{C}^{\alpha} M_{\alpha \beta} C^{\beta}\right)}$,

where $C_{\mu}$ is a complex Grossmann variable. Using this relation, we finally end up with the following relation for the one-loop partition function

$$
\begin{aligned}
Z^{1-\text { loop }}= & \operatorname{det}^{-1 / 2} G_{\alpha \beta} \int D\left[h_{\mu \nu}\right] D\left[C_{\mu}\right] D\left[\bar{C}_{\mu}\right] \\
& \times e^{-\int d^{4} x \sqrt{-g}\left(\mathcal{L}_{2}+\mathcal{L}_{g f}+\mathcal{L}_{g h}\right)},
\end{aligned}
$$

in which the ghost Lagrangian is defined as

$\mathcal{L}_{g h}=\frac{1}{2} \bar{C}^{\alpha} M_{\alpha \beta} C^{\beta}$.

The anticommuting field $C_{\mu}$ is called the Faddeev-Popov ghost.

Let us now York decompose the metric fluctuation $h_{\mu \nu}$ into the transverse-traceless part $A_{\mu \nu}$, the trace part $h$, the helicity- 0 part $\chi$ and the vector part $T$ as

$$
\begin{aligned}
h_{\mu \nu}= & A_{\mu \nu}+\frac{1}{4} g_{\mu \nu} h+2 \nabla_{(\mu} T_{\nu)}+2 \nabla_{\mu} \nabla_{\nu} \chi \\
& -\frac{1}{2} g_{\mu \nu} \nabla_{\alpha} \nabla^{\alpha} \chi,
\end{aligned}
$$

where we have $\nabla^{\mu} A_{\mu \nu}=0=g^{\mu \nu} A_{\mu \nu}$ and $\nabla^{\mu} T_{\mu}=0$. Also, we decompose the ghost field as

$C_{\alpha}=C_{\alpha}^{\perp}+\nabla_{\alpha} C$,

with $\nabla^{\mu} C_{\mu}^{\perp}=0$.

By applying the York decomposition on the second variation of the original perturbed action around the background metric, $\mathcal{L}_{2}$ reads

$$
\begin{aligned}
\frac{1}{2} \mathcal{L}_{2}= & A_{\mu \nu} \mathcal{O}_{A A} A^{\mu \nu}+T_{\mu} \mathcal{O}_{T T} T^{\mu}+\chi \mathcal{O}_{\chi \chi} \chi \\
& +h \mathcal{O}_{h h} h+\chi \mathcal{O}_{h \chi} h .
\end{aligned}
$$

The operators $\mathcal{O}$ can be expressed in terms of some powers of $\square$. The full form of these operators are presented in Appendix B.
We also apply the York decomposition on the other two Lagrangians appeared in (3.14). The gauge-fixing Lagrangian $\mathcal{L}_{g f}$ can be computed as

$$
\mathcal{L}_{g f}=T_{\mu} \mathcal{O}^{\prime}{ }_{T T} T^{\mu}+\chi \mathcal{O}^{\prime}{ }_{\chi \chi} \chi+h \mathcal{O}^{\prime}{ }_{h h} h+h \mathcal{O}^{\prime}{ }_{h \chi} \chi,
$$

where

$$
\begin{aligned}
\mathcal{O}^{\prime}{ }_{T T}= & -\frac{\alpha}{2} \square^{3}+\frac{1}{2}(1-\alpha) \Lambda+\frac{1}{2}(2+\alpha) \Lambda^{2} \square \\
& +\frac{1}{2}(1+\alpha) \Lambda^{3}, \\
\mathcal{O}^{\prime}{ }_{\chi \chi}= & \frac{9}{8} \alpha \gamma \square^{4}+\frac{3}{8}(8 \alpha \gamma-3) \Lambda \square^{3} \\
& +(2 \alpha \gamma-3) \Lambda^{2} \square^{2}-2 \Lambda^{3} \square, \\
\mathcal{O}^{\prime}{ }_{h h}= & \frac{1}{2} \alpha \rho^{2} \gamma \square^{2}-\frac{1}{2} \rho^{2} \Lambda \square, \\
\mathcal{O}^{\prime}{ }_{h \chi}= & -\frac{3}{2} \alpha \rho \gamma \square^{3}+\frac{1}{2} \rho(3-4 \alpha \gamma) \Lambda \square^{2}+2 \rho \Lambda^{2} \square .
\end{aligned}
$$

The ghost Lagrangian can be written as

$\mathcal{L}_{g h}=C^{\perp \mu} \mathcal{O}_{1} C_{\mu}^{\perp}+C \mathcal{O}_{2} C$,

where the operators have the forms

$$
\begin{aligned}
\mathcal{O}_{1}= & -\alpha \square^{2}+\Lambda \square+(1+\alpha) \Lambda^{2}, \\
\mathcal{O}_{2}= & \frac{1}{2}(-\square)\left(\alpha(4 \rho-3) \gamma \square^{2}+(3-4 \rho\right. \\
& \left.-4 \alpha \gamma) \Lambda \square+4 \Lambda^{2}\right) .
\end{aligned}
$$

It can be easily checked that by assuming

$\alpha=1, \quad \rho=-\frac{a_{1}}{2 \Lambda^{2}}, \quad \gamma=-\frac{4 a_{3} \Lambda^{2}}{3 a_{1}}$,

we obtain $\mathcal{O}_{h \chi}+\mathcal{O}^{\prime}{ }_{h \chi}=0$, and therefore the action $\mathcal{L}_{\text {gauged }} \equiv \mathcal{L}_{2}+\mathcal{L}_{g f}$ becomes diagonal. Here, $a_{1}$ and $a_{3}$ are two of the parameters appearing in $\mathcal{O}_{h \chi}$ which are presented explicitly in Appendix B. After some algebra, $\mathcal{L}_{\text {gauged }}$ can be simplified as

$$
\mathcal{L}_{\text {gauged }}=A_{\mu \nu} \mathcal{O}_{A A}^{\prime \prime} A^{\mu \nu}+T_{\mu} \mathcal{O}_{T T}^{\prime \prime} T^{\mu}+h \mathcal{O}_{h h}^{\prime \prime} h+\chi \mathcal{O}_{\chi \chi}^{\prime \prime} \chi,
$$

in which

$$
\begin{aligned}
& \mathcal{O}_{A A}^{\prime \prime}=\left(-\square+m_{A,+}^{2}\right)\left(-\square+m_{A,-}^{2}\right), \\
& \mathcal{O}_{T T}^{\prime \prime}=-(-\square-\Lambda)\left(-\square+m_{T,+}^{2}\right)\left(-\square+m_{T,+}^{2}\right),
\end{aligned}
$$




$$
\begin{aligned}
\mathcal{O}_{h h}^{\prime \prime} & =\left(-\square+m_{h,+}^{2}\right)\left(-\square+m_{h,-}^{2}\right), \\
\mathcal{O}_{\chi \chi}^{\prime \prime} & =(-\square)\left(-\square-\frac{4 \Lambda}{3}\right)\left(-\square+m_{\chi,+}^{2}\right)\left(-\square+m_{\chi,+}^{2}\right) .
\end{aligned}
$$

We have written the explicit expressions of the above masses in Appendix C.

Also, one can easily obtain

$$
\operatorname{det} G_{\alpha \beta}=\operatorname{det}\left(-\square+\frac{(1+\alpha)}{\alpha} \Lambda\right)_{(1)} \operatorname{det}\left(-\square+\frac{\Lambda}{\alpha \gamma}\right)_{(0)}
$$

Obviously for $\alpha=0$ âĂ $\check{N}$ we have $\operatorname{det} G_{\alpha \beta}=1$. The final form of the one-loop partition function of our model is written as

$$
\begin{aligned}
Z^{1 \text {-loop }}= & Z_{\text {gauged }} Z_{g h} Z_{N_{h}} Z_{N_{C}} Z_{N_{b}} \\
= & \operatorname{det}(-\square-\Lambda)_{(1)} \operatorname{det}\left(-\square+\frac{4 \Lambda}{\rho-3}\right)_{(0)} \\
& \times\left[\operatorname{det}(-\square+2 \Lambda)_{(1)} \operatorname{det}\left(-\square+\frac{\Lambda}{\gamma}\right)_{(0)}\right]^{1 / 2} \\
& \times\left[\operatorname{det}\left(-\square+m_{A,+}^{2}\right)_{(2)} \operatorname{det}\left(-\square+m_{A,-}^{2}\right)_{(2)}\right. \\
& \times \operatorname{det}\left(-\square+m_{T,+}^{2}\right)_{(1)} \operatorname{det}\left(-\square+m_{T,-}^{2}\right)_{(1)} \\
& \times \operatorname{det}\left(-\square+m_{h,+}^{2}\right)_{(0)} \operatorname{det}\left(-\square+m_{h,-}^{2}\right)_{(0)} \\
& \left.\times \operatorname{det}\left(-\square+m_{\chi,+}^{2}\right)_{(0)} \operatorname{det}\left(-\square+m_{\chi,-}^{2}\right)_{(0)}\right]^{-1 / 2},
\end{aligned}
$$

where the subscripts (0), (1), and (2) represent the modes of spin $S=0,1,2$, respectively. Note that we have one spin-2 field $A_{\mu \nu}$, three spin-1 fields $T_{\mu}, C_{\mu}^{\perp}$ and $\bar{C}_{\mu}^{\perp}$, and four scalar modes $h, \chi, C$ and $\bar{C}$. Also, the last three partition functions in the first line of the above relation are associated with the decomposition of the fields which are written in Appendix D.

Now, let us consider some special cases of interest of the partition function (3.31).

In the case of the Einstein gravity with the Lagrangian $\mathcal{L}=\kappa^{2}(R-2 \Lambda)$, the appropriate choice of the gauge fixing parameters, in the relations (3.4) and (3.5), for which the scalar part of $\mathcal{L}_{\text {gauged }}$ becomes diagonal would be

$\alpha=0, \quad \rho=-\frac{\kappa^{2}}{2 \Lambda}, \quad \gamma=1$,

and the one-loop partition function (3.31) reduces to

$$
Z_{\text {Einstein }}^{1-\text { loop }}=\sqrt{\frac{\operatorname{det}(-\square-\Lambda)_{(1)}}{\operatorname{det}\left(-\square+\frac{2 \Lambda}{3}\right)_{(2)}}},
$$

which is the expected result [29].

Also, we can recover the result of the one-loop partition function of the conformal gravity theory, first presented in [32]. In this case, the Lagrangian takes the form $\mathcal{L}=\eta C^{\alpha}{ }_{\beta \mu \nu} C_{\alpha}{ }^{\beta \mu \nu}$ which can be converted to a simpler form $\mathcal{L}=\frac{2}{3} \eta\left(3 R_{\mu \nu} R^{\mu \nu}-R^{2}\right)$ using the Gauss-Bonnet theorem. It can be seen that the appropriate choice of the gauge-fixing parameters in this case can be obtained as

$\alpha=0, \quad \rho=0, \quad \gamma=1$,

and the partition function takes the form

$Z^{1 \text {-loop }}=Z_{\text {Einstein }}^{1 \text {-loop }} \sqrt{\frac{\operatorname{det}\left(-\square-\frac{4 \Lambda}{3}\right)_{(0)}}{\operatorname{det}\left(-\square+\frac{4 \Lambda}{3}\right)_{(2)}},}$

which matches with the results of [32], noting that they use $\eta=-2 \hat{a} \breve{A} \breve{N}$ and $\Lambda=-3$. In the case where the Lagrangian takes the form $\mathcal{L}=\kappa^{2}(R-2 \Lambda)+\eta C^{\alpha}{ }_{\beta \mu \nu} C_{\alpha}{ }^{\beta \mu \nu}$, the one-loop partition function becomes

$$
Z^{1 \text {-loop }}=\frac{Z_{\text {Einstein }}^{1 \text {-loop }}}{\sqrt{\operatorname{det}\left(-\square+\frac{4 \Lambda}{3}-\frac{\kappa^{2}}{2 \eta}\right)_{(2)}}} .
$$

As one can see, the spin-0 part disappears from the one-loop partition function. As truly explained in [32], this is related to the fact that the above Lagrangian is not any more conformal invariant.

As a more general case in this category, let us consider the Lagrangian $\mathcal{L}=\kappa^{2}(R-2 \Lambda)-\lambda R^{2}+v R_{\mu \nu} R^{\mu \nu}$, which was first considered in the quantum gravity by Stelle [25]. In this case the appropriate gauge choice can be obtained as

$\alpha=1, \quad \rho=-\frac{\kappa^{2}}{2 \Lambda}, \quad \gamma=\frac{2 \Lambda(v-3 \lambda)}{\kappa^{2}}$,

and the one-loop partition function becomes

$Z^{1 \text {-loop }}=\frac{Z_{\text {Einstein }}^{\text {1-loop }}}{\sqrt{\operatorname{det}\left(-\square-\frac{\kappa^{2}}{2(v-3 \lambda)}\right)_{(0)} \operatorname{det}\left(-\square-\frac{4 \Lambda}{3}-\frac{\kappa^{2}-8 \lambda \Lambda}{v}\right)_{(2)}}}$.

The above expression reduces to (3.34) in the case $v=2 \eta=$ $3 \lambda$.

Now, consider a special case of the $f(R)$ gravity theory, namely $\mathcal{L}=\kappa^{2} R+\eta R^{3}$. This theory admits an AdS background with $\Lambda=-\kappa / 4 \sqrt{\eta}$ provided that $\eta>0$. In this case, the appropriate choice of the gauge-fixing parameters would be

$\alpha=1, \quad \rho=\frac{\kappa^{2}}{\Lambda}, \quad \gamma=-\frac{9}{4}$, 
and the partition function takes the form

$$
Z^{1 \text { loop }}=\frac{Z_{\text {Einstein }}^{\text {1-loop }}}{\sqrt{\operatorname{det}\left(-\square-\frac{4 \Lambda}{9}\right)_{(0)}}} .
$$

At the end, for the theory $\mathcal{L}=\kappa^{2} R+\eta R R_{\mu \nu} R^{\mu \nu}$ discussed in Sect. 2, the appropriate choice of the gauge-fixing parameters is

$\alpha=1, \quad \rho=\kappa^{2}, \quad \gamma=-\frac{5}{2}$,

and the corresponding partition function becomes

$Z^{1 \text {-loop }}=\frac{Z_{\text {Einstein }}^{1 \text {-loop }}}{\sqrt{\operatorname{det}\left(-\square-\frac{2 \Lambda}{5}\right)_{(0)} \operatorname{det}\left(-\square-\frac{10 \Lambda}{3}\right)_{(2)}}}$.

From the above examples, one can deduce that the $f(R)$ gravity theories produce only one spin-2 contribution to the partition function, which is related to the massless graviton of the Einstein's theory. However, adding terms containing $R_{\mu \nu} R^{\mu \nu}$ produces a secondary tensor contribution to the partition function.

\section{The traced heat kernel on $\mathrm{AdS}_{4}$}

This section is devoted to a short explanation of the heat kernel method and the prescription of $[30,31]$, used here to compute the determinants appearing in the one-loop partition functions obtained in the previous section.

We can define the heat kernel of the operator $\square_{(S)}$, for a field with the spin $S$, on a manifold $\mathcal{M}$ between two points $x$ and $y$ as

$K_{a b}^{(S)}(x, y ; t)=\left\langle y, b\left|e^{t \square_{(S)}}\right| x, a\right\rangle$,

where $a$ and $b$ denote Lorentz indices of the field. The operator we are interested in is $\left(\square-m^{2}\right)_{(S)}$ for a spin- $S$ field. The partition function related to the trace of the heat kernel over both the spin indices and space-time is written as

$$
\begin{aligned}
\ln Z_{(S)} & =-\frac{1}{2} \ln \operatorname{det}\left(-\square+m^{2}\right)_{(S)}=-\frac{1}{2} \operatorname{tr} \ln \left(-\square+m^{2}\right)_{(S)} \\
& =\frac{1}{2} \int_{0}^{\infty} \frac{d t}{t} K^{(S)}(t) e^{-t m_{(S)}^{2}}
\end{aligned}
$$

in which the trace of the heat kernel is defined as

$K^{(S)}(t) \equiv \operatorname{tr} e^{t \square_{(S)}}=\int_{\mathcal{M}} d^{d} x \sqrt{-g} \sum_{a} K_{a a}^{(S)}(x, x ; t)$,
Although the computation of the heat kernel in general manifolds is a difficult task even for the scalar operators, for symmetric spaces the use of the harmonic analysis on group manifolds can help us to make the problem tractable. For example for the trace of the heat kernel on the thermal quotient $\mathrm{AdS}_{4}$, the quotient space can be assumed as $H^{4} \simeq S O(4,1) / S O(4)$. For this case the trace of the heat kernel becomes

$K^{(S)}(t)=\frac{\beta}{2 \pi} \sum_{k \in \mathbb{Z}} \sum_{\mathbf{m}} \int_{0}^{\infty} d \lambda \chi_{\lambda, \mathbf{m}}\left(\gamma^{k}\right) e^{t E_{R}^{(S)}}$,

where $E_{R}^{(S)}$ s are the eigenvalues of the spin-s operator on the quotient space $H^{4}, \chi_{\lambda, \mathbf{m}}\left(\gamma^{k}\right)$ is the Harish-Chandra in principle series $S O(4,1), \gamma$ is an element of the thermal quotient of $S^{4}, \beta$ is the inverse temperature $\left(\beta=\frac{1}{k T}\right)$ and $(\lambda, \mathbf{m})$ denotes the principal series representation $[30,31]$.

Now, we need to obtain the eigenvalues for the symmetric transverse traceless tensor that we are interested in. The unitary irreducible representations of $S O(4,1)$ and $S O(4)$ denoted by $R$ and $S$, respectively, are characterized by the following array

$$
\begin{aligned}
& R=\left(m_{1}, m_{2}\right)=\left(i \lambda, m_{2}\right) \quad \text { with } \lambda \in \mathbb{R}, \\
& S=\left(s_{1}, s_{2}\right) \quad \text { with } s_{1} \geqslant s_{2} \geqslant 0,
\end{aligned}
$$

in which $m_{2}$ is a non-negative (half-) integer, and $s_{1}$ and $s_{2}$ are (half-) integer. The eigenvalues of the spin-s operator in the quotient space $H^{4}$ are given by

$-E_{R}^{(S)}=C_{2}(R)-C_{2}(S)$

Here, $C_{2}(R)$ and $C_{2}(S)$ are the Casimirs of the unitary irreducible representation of $S O(4,1)$ and $S O(4)$, respectively, which can be written as

$$
\begin{aligned}
& C_{2}(R)=m^{2}+2 r^{S O(4,1)} \cdot m, \\
& C_{2}(S)=s^{2}+2 r^{S O(4)} \cdot s,
\end{aligned}
$$

where the dot sign denotes the usual Euclidean product, and $r_{i}^{S O(4,1)}=\frac{5}{2}-i, \quad r_{i}^{S O(4)}=2-i, \quad$ for $i=1,2,3$.

Using these relations for the Casimirs, the Eq. (4.6) is obtained as

$-E_{R}^{(S)}=\lambda^{2}+\frac{9}{4}+S$

In the principal series of $S O(4,1)$, the Harish-Chandra character is written as follows

$\chi_{\lambda, \mathbf{m}}\left(\beta, \phi_{1}\right)=\frac{\left(e^{-i \beta \lambda}+e^{i \beta \lambda}\right) \chi_{\mathbf{m}}^{S O(3)}\left(\phi_{1}\right)}{e^{-3 \beta / 2}\left|e^{\beta}-1\right|\left|e^{\beta}-e^{i \phi_{1}}\right|^{2}}$, 
in which $\chi_{\mathbf{m}}^{S O(3)}$ is the character of the $S O(3)$ representation. For the thermal quotient we have $\phi_{1}=0$ and $\chi_{\mathbf{m}}^{S O(3)}(0)=$ $1+2 S$. Substituting the Eqs. (4.8) and (4.9) into (4.4) [30,31], we can find the traced heat kernel as

$K^{(S)}(t)=\frac{\beta(1+2 S)}{8 \sqrt{\pi t}} \sum_{k \in \mathbb{Z}_{+}} \frac{e^{-\frac{k^{2} \beta^{2}}{4 t}-t\left(\frac{9}{4}+S\right)}}{\sinh ^{3} \frac{k \beta}{2}}$.

To prevent the divergence of this equation, we have excluded $k=0$. Finally, substituting this relation into the Eq. (4.2), we can evaluate the partition function for the spin $S$ as follows

$$
\begin{aligned}
\ln Z_{(S)} & =-\frac{1}{2} \ln \operatorname{det}\left(-\square+m^{2}\right)_{(S)} \\
& =(1+2 S) \sum_{k \in \mathbb{Z}_{+}} \frac{\left.e^{-k \beta\left(\frac{3}{2}+\sqrt{\frac{9}{4}+m^{2}+S}\right.}\right)}{\left(1-e^{-k \beta}\right)^{3} k} .
\end{aligned}
$$

Using this relation, we can simply complete the calculation of the one-loop partition function for the cases of our interest with the $\mathrm{AdS}_{4}$ background. The results are presented in the following section.

\section{Thermodynamical implications}

Using the Eq. (4.11), one can obtain the partition function of the general $f\left(R, R_{\mu \nu} R^{\mu \nu}\right)$ gravity theory in a thermal quotient of the $\mathrm{AdS}_{4}$ space. In this section, we consider thermodynamical relations related to the thermal partition function (4.11) in the six special cases discussed in Sect. 3. Note that the one-loop correction to the Helmholtz free energy can be obtained from the one-loop partition function via

$F^{1 \text {-loop }}=-\frac{1}{\beta} \ln Z^{1 \text {-loop }}$.

We start with the case of the Einstein gravity with the Lagrangian $\mathcal{L}=\kappa^{2}(R-2 \Lambda)$. Using the one-loop partition function of this theory [Eq. (3.32)], it can be easily seen that

$\ln Z_{\text {Einstein }}^{1 \text {-loop }}=\frac{1}{2} \ln \operatorname{det}(-\square-\Lambda)_{(1)}-\frac{1}{2} \ln \operatorname{det}\left(-\square+\frac{2 \Lambda}{3}\right)_{(2)}$.

Each of the two spin-contributions in this relation can be evaluated using the Eq. (4.11), which results in the following relation for the free energy of this theory

$$
\begin{aligned}
F_{\text {Einstein }}^{1 \text {-loop }}= & \sum_{k} \frac{e^{-\frac{3}{2} k \beta}}{\left(1-e^{-k \beta}\right)^{3} k \beta}\left[-5 e^{-k \beta \sqrt{\frac{17}{4}+\frac{2 \Lambda}{3}}}\right. \\
& \left.+3 e^{-k \beta \sqrt{\frac{13}{4}-\Lambda}}\right] .
\end{aligned}
$$

In the case of the conformal gravity with $\mathcal{L}=\frac{2}{3} \eta\left(3 R_{\mu \nu}\right.$ $R^{\mu \nu}-R^{2}$ ) by doing a similar calculation, we obtain

$$
\begin{aligned}
F_{\text {Conformal }}^{1 \text {-loop }}= & \sum_{k} \frac{e^{-\frac{3}{2} k \beta}}{\left(1-e^{-k \beta}\right)^{3} k \beta} \\
& \times\left[-5 e^{-k \beta \sqrt{\frac{17}{4}+\frac{2 \Lambda}{3}}}+3 e^{-k \beta \sqrt{\frac{13}{4}-\Lambda}}\right. \\
& \left.+e^{-k \beta \sqrt{\frac{9}{4}-\frac{4 \Lambda}{3}}}-5 e^{-k \beta \sqrt{\frac{17}{4}+\frac{4 \Lambda}{3}}}\right] .
\end{aligned}
$$

One can see that the first two terms in the second line correspond to the ones for the Einstein's theory.

In the case $\mathcal{L}=\kappa^{2}(R-2 \Lambda)+\frac{2}{3} \eta\left(3 R_{\mu \nu} R^{\mu \nu}-R^{2}\right)$ we have

$$
\begin{aligned}
F_{R+\text { Conformal }}^{1 \text {-loop }}= & \sum_{k} \frac{e^{-\frac{3}{2} k \beta}}{\left(1-e^{-k \beta}\right)^{3} k \beta}\left[-5 e^{-k \beta \sqrt{\frac{17}{4}+\frac{2 \Lambda}{3}}}\right. \\
& \left.+3 e^{-k \beta \sqrt{\frac{13}{4}-\Lambda}}-5 e^{-k \beta \sqrt{\frac{17}{4}-\frac{3 k^{2}-8 \eta \Lambda}{6 \eta}}}\right] .
\end{aligned}
$$

For the Stelle's Lagrangian, $\mathcal{L}=\kappa^{2}(R-2 \Lambda)-\lambda R^{2}+$ $v R_{\mu \nu} R^{\mu \nu}$, one can obtain

$$
\begin{aligned}
& F_{\text {Stelle }}^{1 \text {-loo }}=\sum_{k} \frac{e^{-\frac{3}{2} k \beta}}{\left(1-e^{-k \beta}\right)^{3} k \beta} \\
& \times\left[-5 e^{-k \beta \sqrt{\frac{17}{4}+\frac{2 \Lambda}{3}}}+3 e^{-k \beta \sqrt{\frac{13}{4}-\Lambda}}\right. \\
& \left.-e^{-k \beta \sqrt{\frac{9}{4}-\frac{\kappa^{2}}{2(v-3 \lambda)}}}-5 e^{-k \beta \sqrt{\frac{17}{4}-\frac{4 \Lambda}{3}-\frac{\kappa^{2}-8 \lambda \Lambda}{v}}}\right] .
\end{aligned}
$$

For the Lagrangian $\mathcal{L}=\kappa^{2} R+\eta R^{3}$, the free energy can be obtained as

$$
\begin{aligned}
F_{\kappa^{2} R+\eta R^{3}}^{1 \text {-loop }}= & \sum_{k} \frac{e^{-\frac{3}{2} k \beta}}{\left(1-e^{-k \beta}\right)^{3} k \beta}\left[-5 e^{-k \beta \sqrt{\frac{17}{4}+\frac{2 \Lambda}{3}}}\right. \\
& \left.+3 e^{-k \beta \sqrt{\frac{13}{4}-\Lambda}}-e^{-k \beta \sqrt{\frac{9}{4}-\frac{4 \Lambda}{9}}}\right] .
\end{aligned}
$$

The last example is $\mathcal{L}=\kappa^{2} R+\eta R R_{\mu \nu} R^{\mu \nu}$ with the free energy

$$
\begin{aligned}
F_{\kappa^{2} R+\eta R R_{\mu \nu} R^{\mu \nu}}^{1 \text {-loop }}= & \sum_{k} \frac{e^{-\frac{3}{2} k \beta}}{\left(1-e^{-k \beta}\right)^{3} k \beta} \\
& \times\left[-5 e^{-k \beta \sqrt{\frac{17}{4}+\frac{2 \Lambda}{3}}}+3 e^{-k \beta \sqrt{\frac{13}{4}-\Lambda}}\right. \\
& \left.-e^{-k \beta \sqrt{\frac{9}{4}-\frac{2 \Lambda}{5}}}-5 e^{-k \beta \sqrt{\frac{17}{4}-\frac{10 \Lambda}{3}}}\right] .
\end{aligned}
$$




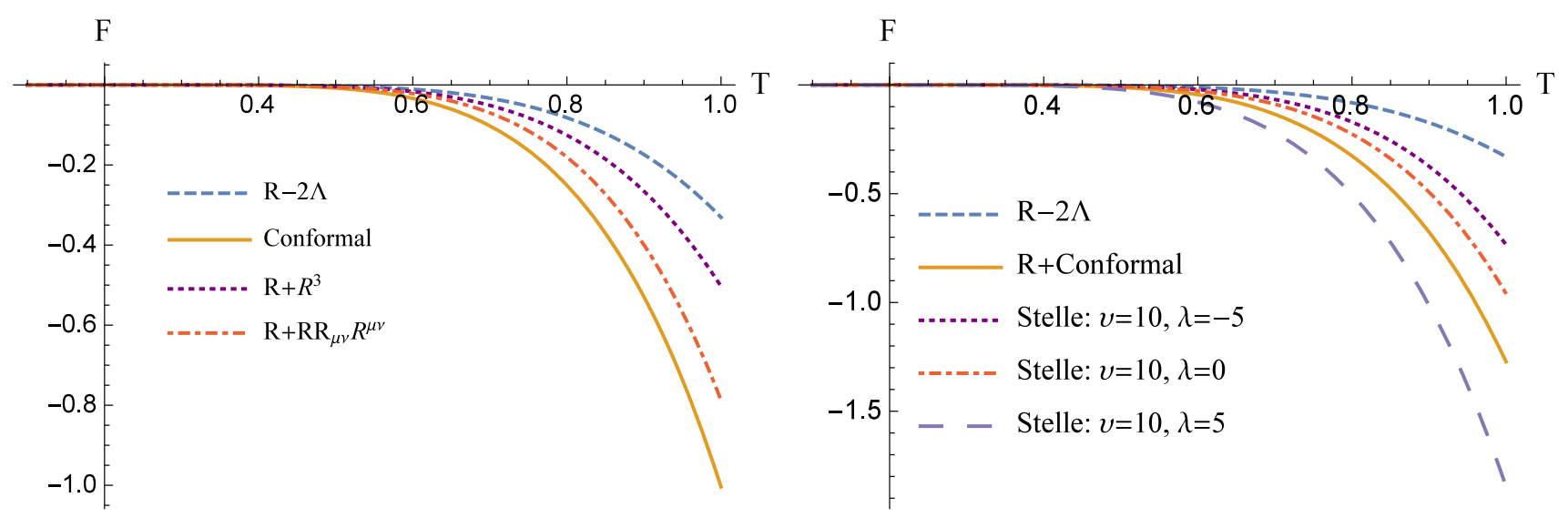

Fig. 1 One-loop correction to the free energy in special cases of $f\left(R, R_{\mu \nu} R^{\mu v}\right)$. We have assumed $\Lambda=-1$ and $\eta=1$. The values for $v$ and $\lambda$ are shown in the figure

One can, moreover, obtain the one-loop quantum correction to the entropy as

$S^{1 \text { loop }}=-\frac{\partial F^{1-\text { loop }}}{\partial T}$,

where $T=k \beta$ is the temperature. Note that in this paper we have considered the $\mathrm{AdS}_{4}$ space as the background. The entropy and temperature of the $\mathrm{AdS}_{4}$ are zero, i.e., the background values for the thermodynamical quantities are zero. In Figs. 1 and 2, we have plotted the above expressions for the one-loop correction to the free energy as well as the entropy, for all the sample theories. One can see from the figures that the correction of the free energy in all cases are negative, indicating that the quantum correction decreases the background values of free energy. The smallest change in the free energy is associated with the Einstein-Hilbert action. Any modifications to the Einstein-Hilbert action increases the one-loop quantum correction to the free energy. From the figures, one can deduce that by increasing the value for $\lambda$ in the Lagrangian $\mathcal{L}=\kappa^{2}(R-2 \Lambda)-\lambda R^{2}+v R_{\mu \nu} R^{\mu \nu}$, the quantum correction to the free energy is increased.

The quantum correction to the entropy is very similar to the effects of the free energy, however in a reverse direction due to the minus sign in (5.7). This means that the quantum corrections to the entropy increase the background value.

\section{Conclusions and final remarks}

In this paper we have computed the partition function at the one-loop level for a modified theory of gravity containing a function of both the Ricci scalar and the square of the Ricci tensor. Studying this theory can be very informative in the context of quantum gravity, since it can be thought of as a generalization of many other important modified gravity theories. For our purpose we have used the usual Faddeev-

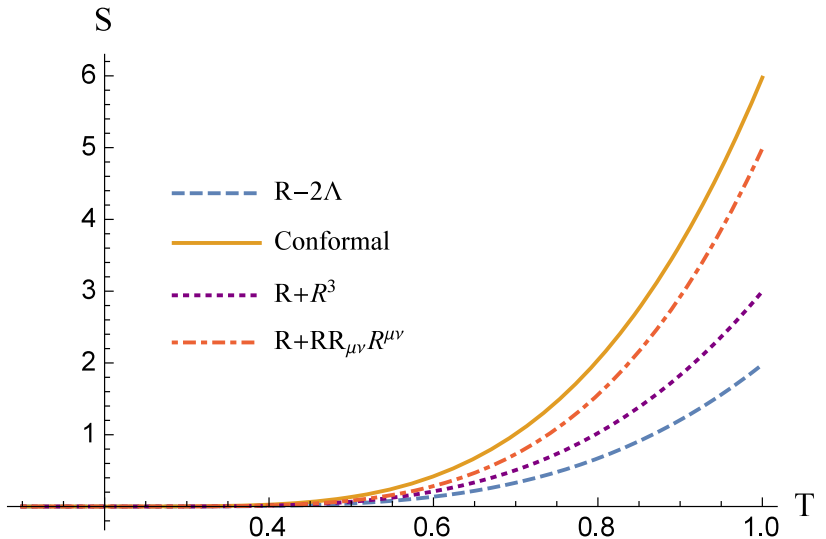

Fig. 2 One-loop correction to the entropy in four special cases of $f\left(R, R_{\mu \nu} R^{\mu \nu}\right)$. We have assumed $\Lambda=-1$

Popov and De Witt method to find the total partition function at the one-loop level over a constant curvature background metric. Then, using the York decomposition we were able to calculate this partition function in terms of the determinants of scalar, vector and tensor modes of the metric tensor. We have moreover showed that the theory chosen here has $\mathrm{AdS}_{4}$ solution by presenting an example in the paper. Therefore, we were able to find the one-loop partition function of our theory over the $\mathrm{AdS}_{4}$ background, which is of great interest in other contexts, for instance in the AdS/CFT correspondence. In this regard, we have also presented the calculated oneloop partition function over the $\mathrm{AdS}_{4}$ solution for 6 different gravity theories at the end of Sect. 3 . Then, we have used the heat kernel method to compute the determinants of different operators in the partition function. The heat kernel representation is interesting since it shows a way to construct a traced heat kernel of the $\mathrm{AdS}_{4}$ background. We have used this procedure to compute the one-loop thermal partition function of the theory over the $\mathrm{AdS}_{4}$ space. This should be considered as a one-loop correction to the background partition 
function of the dual CFT theory. We have then straightforwardly computed the one-loop quantum corrections to both the Helmholtz free energy and the entropy of the theories we are interested in. It is interesting that for all theories we have considered in this paper, the one-loop quantum corrections increase the entropy from its value at the background.

As a final remark, one should note that the above calculations can be done equivalently for the dS space. For more details see [26]. However, the traced heat kernel is only calculated for the AdS space. Thus, the thermodynamical considerations can only be done over the AdS background.

While this work was in progress, the preprint [34] appeared on arXiv in which the authors have done a similar calculation as is presented in this manuscript and obtain the one-loop divergences of the same theory. However, our calculations are different in the way that besides using a different approach for fixing the gauge, we are also interested in the thermodynamical implications of the theory over thermal quotient of the $\mathrm{AdS}_{4}$ space which is not the scope of [34]. In [34], the authors have made a linear transformation in the scalar sector to make it diagonal and then used the standard GR gauge choice. However, we have used the most general gauge choice and then chosen the parameters in such a way that the scalar sector becomes diagonal.

Open Access This article is distributed under the terms of the Creative Commons Attribution 4.0 International License (http://creativecomm ons.org/licenses/by/4.0/), which permits unrestricted use, distribution, and reproduction in any medium, provided you give appropriate credit to the original author(s) and the source, provide a link to the Creative Commons license, and indicate if changes were made.

Funded by SCOAP ${ }^{3}$.

\section{A: Derivation of Eq. (3.8)}

After transforming $h_{\mu}^{\alpha}$ and $h$ in the Eq. (3.4) according to (3.2), we obtain

$\delta \xi^{\mu}=\square \epsilon^{\mu}+\nabla_{\nu} \nabla^{\mu} \epsilon^{\nu}-2\left(\rho+\frac{1}{4}\right) \nabla^{\mu} \nabla_{\nu} \epsilon^{\nu}$.

Now changing the ordering of the covariant derivatives in the second term of the right-hand side of (A.1), one obtains

$$
\begin{aligned}
\delta \xi^{\mu} & =\square \epsilon^{\mu}+\nabla^{\mu} \nabla_{\nu} \epsilon^{\nu}+R_{\nu}^{\mu} \epsilon^{\nu}-2\left(\rho+\frac{1}{4}\right) \nabla^{\mu} \nabla_{\nu} \epsilon^{\nu} \\
& =\square \epsilon^{\mu}+R_{\nu}^{\mu} \epsilon^{\nu}-2\left(\rho-\frac{1}{4}\right) \nabla^{\mu} \nabla_{\nu} \epsilon^{\nu},
\end{aligned}
$$

which leads to the Eq. (3.8) after differentiation with respect to $\epsilon^{v}$.

\section{B: Operators in the second order variation of the action}

$$
\begin{aligned}
\mathcal{O}_{A A}= & f_{X} \square^{2}+\left(f_{R}+\frac{2 \Lambda}{3} f_{X}\right) \square \\
& +\left(f-\frac{4}{9} \Lambda\left(6 f_{R}+11 \Lambda f_{X}\right)\right) . \\
\mathcal{O}_{T T}= & 2 c_{1}(\square+\Lambda) . \\
\mathcal{O}_{x \chi}= & \frac{3}{2}\left(2 f_{X}+3 c_{2}\right) \square^{4}-\frac{1}{2}\left(3 f_{R}-8 \Lambda\left(f_{X}+3 c_{2}\right)\right) \square^{3} \\
& +\left(3 f-8 \Lambda f_{R}+4 \Lambda^{2}\left(2 c_{2}-3 f_{X}\right)\right) \square^{2}-4 \Lambda c_{1} \square .
\end{aligned}
$$

$$
\begin{aligned}
\mathcal{O}_{h h}= & \frac{3}{8}\left(2 f_{X}+3 c_{2}\right) \square^{2}-\frac{1}{8}\left(3 f_{R}-8 \Lambda\left(f_{X}+3 c_{2}\right)\right) \square \\
& +\frac{1}{4}\left(f-4 \Lambda f_{R}+4 \Lambda^{2}\left(2 c_{2}-f_{X}\right)\right) . \\
\mathcal{O}_{h \chi}= & a_{3} \square^{3}+a_{2} \square^{2}+a_{1} \square,
\end{aligned}
$$

where

$a_{1}=2 \Lambda\left(f_{R}-4 \Lambda c_{2}\right)$,

$a_{2}=\frac{1}{2}\left[3 f_{R}-8 \Lambda\left(f_{X}+3 c_{2}\right)\right]$,

$a_{3}=-\frac{3}{2}\left(2 f_{X}+3 c_{2}\right)$,

and

$c_{1}=2 \Lambda\left(f_{R}+2 \Lambda f_{X}\right)-f$,

$c_{2}=f_{R R}+4 \Lambda\left(f_{X R}+\Lambda f_{X X}\right)$.

One can easily check that the following relation holds

$\frac{3}{4 \Lambda} a_{1}-a_{2}+\frac{4 \Lambda}{3} a_{3}=0$.

\section{C: The masses}

$$
\begin{aligned}
m_{A, \pm}^{2}= & \frac{1}{6 f_{X}}\left(-3 f_{R}-2 \Lambda f_{X}\right. \\
& \left. \pm 3 \sqrt{f_{R}^{2}-4 f f_{X}+12 \Lambda f_{R} f_{X}+20 \Lambda^{2} f_{X}^{2}}\right) \\
m_{T, \pm}^{2}= & \frac{1}{2}\left(\Lambda \pm \sqrt{9 \Lambda^{2}+16 \Lambda f_{R}-8 f+32 \Lambda^{2} f_{X}}\right) . \\
m_{h, \pm}^{2}= & \left\{12 f_{X}+8\left[2 \rho^{2} \gamma+9 \Lambda\left(f_{X X} \Lambda+f_{X R}\right)\right]+18 f_{R R}\right\}^{-1} \\
& \times\left\{\left[8 \left(4 \Lambda \left(\Lambda \left(f_{X}-2\left(4 \Lambda \left(f_{X X} \Lambda\right.\right.\right.\right.\right.\right.\right. \\
& \left.\left.\left.\left.\left.+f_{X R}\right)+f_{R R}\right)\right)+f_{R}\right)-f\right)
\end{aligned}
$$




$$
\begin{aligned}
\times & \left(6 f_{X}+4\left(2 \rho^{2} \gamma+9 \Lambda\left(f_{X X} \Lambda+f_{X R}\right)\right)+9 f_{R R}\right) \\
+ & \left(3 f_{R}-8 \Lambda\left(-\rho^{2}+f_{X}+12 \Lambda\left(f_{X X} \Lambda+f_{X R}\right)\right.\right. \\
& \left.\left.\left.+3 f_{R R}\right)\right)^{2}\right]^{1 / 2} \\
& -8 \Lambda\left(-\rho^{2}+f_{X}+12 \Lambda\left(f_{X X} \Lambda\right.\right. \\
& \left.\left.\left.+f_{X R}\right)+3 f_{R R}\right)+3 f_{R}\right\} . \\
m_{\chi, \pm}^{2}= & \left\{8 f_{X}+6\left[\gamma+8 \Lambda\left(f_{X X} \Lambda+f_{X R}\right)+2 f_{R R}\right]\right\}^{-1} \\
& \times\left\{-4 \gamma \Lambda+\left[\left(\Lambda \left(4 \gamma+32 f_{X X} \Lambda^{2}+32 f_{X R} \Lambda\right.\right.\right.\right. \\
& \left.\left.+8 f_{R R}-3\right)-2 f_{R}\right)^{2} \\
& -16\left(f-\Lambda\left(4 f_{X} \Lambda+2 f_{R}+\Lambda\right)\right)\left(4 f_{X}+3(\gamma\right. \\
+ & \left.\left.\left.8 \Lambda\left(f_{X X} \Lambda+f_{X R}\right)+2 f_{R R}\right)\right)\right]^{1 / 2} \\
- & \left.32 f_{X X} \Lambda^{3}+2 f_{R}-32 f_{X R} \Lambda^{2}-8 f_{R R} \Lambda+3 \Lambda\right\}
\end{aligned}
$$

\section{D: The decomposition determinants}

In this section we compute $Z_{N_{h}}$ appeared in the expression of the partition function. The determinants $Z_{N_{C}}$ and $Z_{N_{b}}$ can be obtained in a similar way. First note that

$1=\int D\left[h_{\mu \nu}\right] e^{-\int d^{4} x \sqrt{-g} h_{\mu \nu} h^{\mu \nu}}$.

We want to compute the Jacobian of the transformation which is in the form

$$
D\left[h_{\mu \nu}\right]=Z_{N_{h}} D\left[A_{\mu \nu}\right] D\left[T_{\mu}\right] D[\chi] D[h] .
$$

Inserting the relation (3.16) into (D.1) and integrating by parts, one can obtain

$$
\begin{aligned}
1= & \int Z_{N_{h}} D\left[A_{\mu \nu}\right] D\left[T_{\mu}\right] D[\chi] D[h] \\
& \times \exp \left\{-\int d^{4} x \sqrt{-g}\left[A_{\mu \nu} A^{\mu \nu}+\frac{1}{4} h^{2}\right.\right. \\
& \left.\left.+2 T_{\mu}(-\square-\Lambda) T^{\mu}+3 \chi(-\square)\left(-\square-\frac{4 \Lambda}{3}\right) \chi\right]\right\} .
\end{aligned}
$$

Using the Gaussian integral for the fields $A_{\mu \nu}, T_{\mu}, h$ and $\chi$, one can obtain $Z_{N_{h}}$ as

$Z_{N_{h}}=\left[\operatorname{det}(-\square-\Lambda)_{(1)} \operatorname{det}(-\square)_{(0)} \operatorname{det}\left(-\square-\frac{4 \Lambda}{3}\right)_{(0)}\right]^{1 / 2}$.
Similarly, one can find

$Z_{N_{C}}=\operatorname{det}^{-1}(-\square)_{(0)}$.

\section{References}

1. A. De Felice, S. Tsujikawa, Living Rev. Relativ. 13, 3 (2010). Please note that Refs.[1-4, 6, 7, 13] were split and renumbered sequentially. Please check and confirm

2. T.P. Sotiriou, V. Faraoni, Rev. Mod. Phys. 82, 451 (2010)

3. S. Nojiri, S.D. Odintsov, Phys. Rep. 505, 59 (2011). arXiv: 1011.0544

4. S. Nojiri, S.D. Odintsov, V.K. Oikonomou, Phys. Rep. 692, 1 (2017). arXiv:1705.11098

5. G't Hooft, M. Veltman, Ann. Inst. Henri Poincare 20, 69 (1974)

6. S. Deser, P. van Nieuwenhuizen, Phys. Rev. D 10, 401 (1974)

7. S. Deser, P. van Nieuwenhuizen, Phys. Rev. D 10, 411 (1974)

8. S. Deser, P. van Nieuwenhuizen, H.S. Tsao, Phys. Rev. D 10, 3337 (1974)

9. B. Utiyama, B. DeWitt, J. Math. Phys. 3, 608 (1962)

10. S. Deser, in Gauge Theories and Modem Field Theory, Proceedings of the Boston Conference, 1975, ed. by A. Axnowitt, P. Nath (MIT, Cambridge, 1976)

11. I.G. Avramidi, A.O. Barvinsky, Phys. Lett. B 159, 269 (1985)

12. M.S. Ruf, C.F. Steinwachs, Phys. Rev. D 97, 044049 (2018)

13. M.S. Ruf, C.F. Steinwachs, Phys. Rev. D 97, 044050 (2018)

14. G. Cognola, E. Elizalde, S. Nojiri, S.D. Odintsov, S. Zerbini, JCAP 02, 010 (2005)

15. G. Cognola, S. Zerbini, J. Phys. A Math. Theor. 45, 374014 (2012). arXiv:1203.5032 [gr-qc]

16. P. Horava, Phys. Rev. D 79, 084008 (2009)

17. E. Kiritsis, G. Kofinas, Nucl. Phys. B 821, 467 (2009)

18. P. Horava, Phys. Rev. Lett. 102, 161301 (2009)

19. G. Amelino-Camelia, Living Rev. Relativ. 16, 5 (2013)

20. D. Orlando, S. Reffert, Phys. Rev. D 80, 041501 (2009)

21. E.N. Saridakis, Eur. Phys. J. C 67, 229 (2010)

22. Z. Haghani, T. Harko, H. R. Sepangi, S. Shahidi. arXiv:1404.7689 [gr-qc]

23. T. Jacobson, D. Mattingly, Phys. Rev. D 64, 024028 (2001)

24. D. Blas, O. Pujolas, S. Sibiryakov, JHEP 10, 029 (2009)

25. K. Stelle, Phys. Rev. D 16, 953 (1977)

26. K. Bamba, G. Cognola, S.D. Odintsov, S. Zerbini, Phys. Rev. D 90, 023525 (2014). arXiv:1404.4311 [gr-qc]

27. J.M. Maldacena, Adv. Theor. Math. Phys. 2, 231 (1998)

28. I.L. Buchbinder, S. Odintsov, L. Shapiro, Effective Action in Quantum Gravity (CRC Press, Boca Raton, 1992)

29. E.S. Fradkin, A.A. Tseytlin, Nucl. Phys. B 234, 472 (1984)

30. R. Gopakumar, R. Kumar Gupta, S. Lal, JHEP 11, 010 (2011)

31. J.R. David, M.R. Gaberdiel, R. Gopakumar, JHEP 04, 125 (2010)

32. M. Irakleidou, I. Lovrekovic, Phys. Rev. D 93, 104043 (2016). arXiv: 1512.07130 [hep-th]

33. M. Peskin, D.V. Schroeder, An Introduction to Quantum Field Theory (Addison-Wesley, Boston, 1995)

34. N. Ohta, R. Percacci, A. D. Pereira, Phys. Rev. D 97, 104039 (2018). arXiv:1804.01608 [hep-th] 